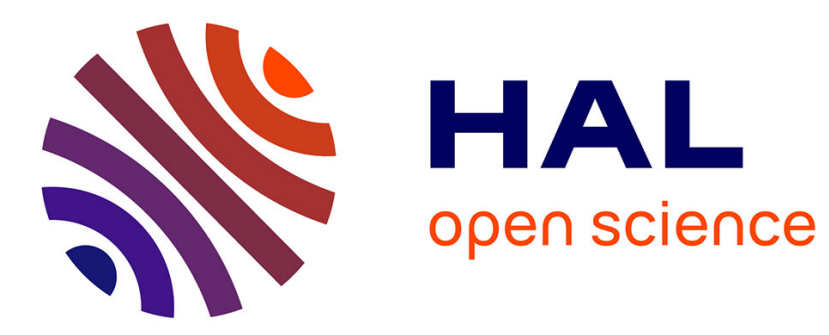

\title{
Global asymptotic stability of a genetic negative feedback loop with an affine control \\ Lucie Chambon, Jean-Luc Gouzé
}

\section{To cite this version:}

Lucie Chambon, Jean-Luc Gouzé. Global asymptotic stability of a genetic negative feedback loop with an affine control. CDC 2019 - 58th IEEE Conference on Decision and Control, Dec 2019, Nice,

France. hal-02418431

\author{
HAL Id: hal-02418431 \\ https://hal.inria.fr/hal-02418431
}

Submitted on 18 Dec 2019

HAL is a multi-disciplinary open access archive for the deposit and dissemination of scientific research documents, whether they are published or not. The documents may come from teaching and research institutions in France or abroad, or from public or private research centers.
L'archive ouverte pluridisciplinaire HAL, est destinée au dépôt et à la diffusion de documents scientifiques de niveau recherche, publiés ou non, émanant des établissements d'enseignement et de recherche français ou étrangers, des laboratoires publics ou privés. 


\title{
Global asymptotic stability of a genetic negative feedback loop with an affine control
}

\author{
Lucie Chambon and Jean-Luc Gouzé
}

\begin{abstract}
Genetic negative feedback loops are essential and recurrent biological motifs. They are traditionally described with $\mathrm{N}$-dimensional competitive dynamical systems, composed of highly non-linear Hill functions. The stability property of their unique steady state usually determines the global dynamical behavior: homeostasis under stability or emergence of oscillations. When homeostasis conditions are disrupted, undesired oscillations can emerge and may lead to various diseases. This paper presents a classical affine control strategy that is able to stabilize the unstable steady state of the disrupted system and suppress undesired oscillations. For biological purpose, this control is designed as simple as possible in order to reduce the use of devices and the complexity of the biological set-up. For this reason, the control law only depends on the measurement of a unique gene and only acts on its own expression. Due to the complexity of this controlled dynamical system, a new methodology, based on the construction of successive hyperrectangles of the state space that act as Lyapunov function level-sets, is proposed in order to prove global convergence and global stability results. Despite its apparent simplicity, this affine control law is shown to globally stabilize the disrupted system and recover homeostasis.
\end{abstract}

\section{INTRODUCTION}

Negative feedback loops are key building blocks in living organisms [8]. They are known to be involved in two biological behaviors: homeostasis (this is the capacity of an organism to keep an internal parameter constant regardless of any external perturbation) and biological oscillators (such as the circadian clock or the cell cycle). It happens that a negative circuit designed for homeostasis becomes disrupted, leading to the emergence of undesired oscillations. This loss of vital homeostasis, called dyshomeostasis, can cause disorders or diseases. As an example, in healthy conditions, the protein p53 involved in apoptosis is tightly controlled by the protein Mdm2 through a negative feedback loop. This system prevents extreme concentrations of $\mathrm{p} 53$, responsible for neurodegenerative diseases [10] and embryonic lethality. These realities provide evidence that finding biologically relevant control strategies in order to compensate and understand these disruptions is of high interest.

In biology, many control tools have been developed in the recent years in order to tackle these kind of problems. The

This work was supported by Région PACA and by the French Government (National Research Agency, ANR) through the "Investments for the Future" LABEX SIGNALIFE: program reference ANR-11-LABX-0028-01.

L. Chambon is with Université Côte d'Azur, Inria, INRA, CNRS, Sorbonne Université, Biocore Team, Sophia Antipolis, France lucie.chambondinria.fr

J.L. Gouzé is with Université Côte d'Azur, Inria, INRA, CNRS, Sorbonne Université, Biocore Team, Sophia Antipolis, France jean-luc.gouzedinria.fr use of inducer molecules [6], the modification of environmental conditions such as the temperature or the osmotic pressure [11], as well as really recent techniques such as Optogenetics that uses photo-sensitive molecules [7], are famous examples of biological means of control. However, these experiments are always tedious, and need really specific and expensive devices in order to measure the system and design the inputs. For this reason, it is important to keep in mind these biological constraints when designing a control strategy in a biological context, and propose a control as simple, robust and efficient as possible.

These biological realities have led to the construction of new tools and frameworks in the field of dynamical systems and control theory. A famous example is the field of monotone dynamical systems [9] whose whole theory has been largely applied to biological problems. Specifically, population dynamics and biological interaction networks are well explained with cooperative and competitive systems. Due to order-preserving, these systems have really nice properties regarding convergence and stability. More recently, many of these results have been extended to monotone control systems and more precisely monotone SISO (single-inputsingle-output) systems [3]. Under appropriate conditions, these feedback systems are able to recover the same global convergence properties than simple monotone systems.

In this context, this paper presents a $N$-dimensional competitive dynamical model for a genetic negative feedback loop that displays dyshomeostasis, leading to the emergence of undesired oscillations. This typically happens when its unique steady state is unstable. A simple affine control law is designed in order to stabilize the system and recover homeostasis properties. The control strategy only depends on the measurement of a unique gene and only acts on a unique genetic interaction. From a biological point of view, this simple control guarantees a minimal biological set-up and helps reducing the complexity of measurement and control devices. However, the resulting controlled system loses its competitive property. Hence, a new methodology is presented in order to prove that this simple control law stabilizes the unstable steady state. The proof is based on the construction of successive repelling nested hyperrectangles that act as Lyapunov function level-sets.

Section II presents the competitive dynamical model for the $N$-dimensional negative feedback loop with an unstable steady state. The control strategy is introduced in section III and the new methodology about global convergence and stability is presented in section IV. In section V-A, the 
controlled system is shown to be composed of sigmoid functions, a key property in order to apply the results from section IV. Finally, section V-B shows that the affine control strategy leads to global stabilization of the steady state and allows homeostasis recovery.

\section{NEGATIVE FEEDBACK LOOP MODEL}

With $N$ components, a genetic negative feedback loop is described by the following system [5]:

$$
\left\{\begin{array}{l}
\dot{x}_{1}\left(x_{1}, x_{N}\right)=\kappa_{01}+\kappa_{1} \mathrm{~h}^{-}\left(x_{N}, \theta_{N}, n_{N}\right)-\gamma_{1} x_{1}, \\
\dot{x}_{i}\left(x_{i}, x_{i-1}\right)=\kappa_{0 i}+\kappa_{i} \mathrm{~h}^{+}\left(x_{i-1}, \theta_{i-1}, n_{i-1}\right)-\gamma_{i} x_{i},
\end{array}\right.
$$

$\forall i \in\{2, \ldots, N\}$, where $\mathrm{h}^{+}(x, \theta, n)=x^{n} /\left(\theta^{n}+x^{n}\right)$ and $\mathrm{h}^{-}(x, \theta, n)=1-\mathrm{h}^{+}(x, \theta, n)$ are Hill functions. They are sigmoid functions and respectively model activation and repression between variables with threshold $\theta>0$ and steepness $n \geq 2$. The parameter $n$ is an integer greater or equal to 2 that represents the number of transcription factors that are involved in the interaction. In addition, biological components are produced with a basal rate $\kappa_{0 i} \geq 0$, degraded with a rate $\gamma_{i}>0$ and experience interaction with intensity $\kappa_{i}>0$. A graph of this system is illustrated in Fig. 1 .

Property 1: System (1) has a unique steady state, that will be called $\bar{x}$ throughout the paper.

Proof: It is possible to show that $x=\left(x_{1}, \ldots, x_{N}\right)=$ $\left(H_{1}\left(x_{N}\right), H_{2}\left(x_{1}\right), \ldots, H_{N}\left(x_{N-1}\right)\right)$ is a steady state of system (1) if and only if $x_{1}$ is a fixed point of the function $S_{1}(x)=$ $H_{1} \circ H_{N} \circ H_{N-1} \circ \ldots \circ H_{3} \circ H_{2}(x)$, where

$$
\left\{\begin{array}{l}
H_{1}(x)=\frac{\kappa_{01}+\kappa_{1} \mathrm{~h}^{-}\left(x, \theta_{N}, n_{N}\right)}{\gamma_{1}}, \\
H_{i}(x)=\frac{\kappa_{0 i}+\kappa_{i} \mathrm{~h}^{+}\left(x, \theta_{i-1}, n_{i-1}\right)}{\gamma_{i}} \quad \forall i \in\{2, \ldots, N\} .
\end{array}\right.
$$

As $S_{1}(x)$ is a strictly monotonically decreasing function on $\mathbb{R}^{+}$, it has a unique positive fixed point.

Depending on the parameters, this negative loop displays two main behaviors: homeostasis if $\bar{x}$ is globally asymptotically stable (GAS) or emergence of sustained oscillations [5]. It is supposed here that this system shows sustained oscillations, that are considered undesired as explained in the introduction. The goal of this paper is to prove that a simple affine control strategy is able to globally stabilize the steady state $\bar{x}$ in order to recover homeostasis.

Remark 1: By studying the vector field, it is straightforward that system (1) is a priori bounded: $\left.x_{1} \in\right] \kappa_{01} / \gamma_{1},\left(\kappa_{01}+\right.$ $\left.\left.\kappa_{1}\right) / \gamma_{1}\right], x_{i} \in\left[\kappa_{0 i} / \gamma_{i},\left(\kappa_{0 i}+\kappa_{i}\right) / \gamma_{i}\left[\forall i \in\{2, \ldots, N\}\right.\right.$, and $\bar{x}_{i} \in$ ]$\kappa_{0 i} / \gamma_{i},\left(\kappa_{0 i}+\kappa_{i}\right) / \gamma_{i}[\forall i \in\{1, \ldots, N\}$. It follows that this system is positive: all the results will concern the invariant positive orthant $\mathbb{R}_{+}^{n}$.

\section{CONTROL LAW}

The selected control consists of a classical affine law $u\left(x_{1}\right)=-\alpha\left(x_{1}-\bar{x}_{1}\right)+1$, with $\alpha>0$, that only depends

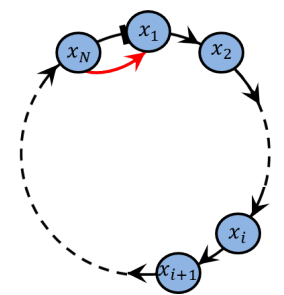

Fig. 1. Graph of the uncontrolled system (1) in black. For the controlled system (3), the graph is the same with the additional red arrow. In this case, the influence of $x_{N}$ on $x_{1}$ is not fixed: it can either activate or inhibit its production.

on the measurement of the first gene $x_{1}$ and acts on its production as the following:

$$
\left\{\begin{array}{l}
\dot{x}_{1}\left(x_{1}, x_{N}\right)=\kappa_{01}+u\left(x_{1}\right) \kappa_{1} \mathrm{~h}^{-}\left(x_{N}, \theta_{N}, n_{N}\right)-\gamma_{1} x_{1}, \\
\dot{x}_{i}\left(x_{i}, x_{i-1}\right)=\kappa_{0 i}+\kappa_{i} \mathrm{~h}^{+}\left(x_{i-1}, \theta_{i-1}, n_{i-1}\right)-\gamma_{i} x_{i}
\end{array}\right.
$$

$\forall i \in\{2, \ldots, N\}$. This law depends on the estimation error $\left(x_{1}-\bar{x}_{1}\right)$ and can take both positive and negative values (see Fig. 1). The constant +1 has been fixed in order to preserve $\bar{x}$ as a steady state:

Property 2: The steady state $\bar{x}$ of system (1) is also the unique steady state of system (3).

Proof: As for the proof of Property 1, it is possible to show that $x=\left(x_{1}, \ldots, x_{N}\right)=\left(H_{1 \alpha}\left(x_{N}\right), H_{2}\left(x_{1}\right), \ldots, H_{N}\left(x_{N-1}\right)\right)$ is a steady state of system (3) if and only if $x_{1}$ is a fixed point of the function $S_{1 \alpha}(x)=H_{1 \alpha} \circ H_{N} \circ H_{N-1} \circ \ldots \circ H_{3} \circ H_{2}(x)$, where

$$
H_{1 \alpha}(x)=\frac{\kappa_{01}+\kappa_{1} \mathrm{~h}^{-}\left(x, \theta_{N}, n_{N}\right)\left(1+\alpha \bar{x}_{1}\right)}{\gamma_{1}+\alpha \kappa_{1} \mathrm{~h}^{-}\left(x, \theta_{N}, n_{N}\right)} .
$$

From the properties of Hill functions and Remark 1 it is easy to show that $H_{1 \alpha}(x)$, and therefore $S_{1 \alpha}(x)$, are strictly monotonically decreasing on $\mathbb{R}^{+}$. As a consequence, $S_{1 \alpha}(x)$ has a unique fixed point, and therefore system (3) has a unique steady state on $\mathbb{R}^{+}$. To conclude, it is easy to check that the steady state $\bar{x}$ of system (1) is a steady state of system (3)

Under good conditions, it may be possible to show that this affine control law is able to locally stabilize $\bar{x}$ with classical control theory [4]. However, the goal here is more challenging, and consists in finding conditions on $\alpha$ such that $\bar{x}$ becomes GAS.

Next section presents a new methodology and gives sufficient conditions on system (3) such that the steady state $\bar{x}$ becomes GAS.

\section{A NEW METHODOLOGY FOR GLOBAL RESULTS}

This methodology is similar to the one developed in [2] or in [3] for SISO monotone dynamical systems. However, system (3) does not have the appropriate conditions and structure in order to apply the results presented in these two previous studies. The new methodology consists in building 
consecutive repelling hyperrectangles of the state space. The condition on system (3) ensures that these hyperrectangles shrink in all directions around $\bar{x}$, so that the dynamics is trapped and cannot do anything else than converging towards $\bar{x}$. Basically, this technique is similar to the construction of Lyapunov function level-sets. In the end, the determination of the global stability of $\bar{x}$ will be directly related to the number of fixed points of the following specific function called $F_{1 \alpha}(x)$ :

Definition 1: $\forall i \in\{1, \ldots, N\}, S_{i \alpha}(x)=H_{i} \circ H_{i-1} \circ \ldots \circ H_{1 \alpha} \circ$ $H_{N} \circ H_{N-1} \circ \ldots \circ H_{i+2} \circ H_{i+1}(x)$, where $i-1=N$ for $i=1$. Then, $\forall i \in\{1, \ldots, N\}$ the function $F_{i \alpha}(x)$ is defined as the composition of $S_{i \alpha}(x)$ with itself: $F_{i \alpha}(x)=S_{i \alpha} \circ S_{i \alpha}(x)$.

The main result is the following:

Theorem 1: If the function $F_{1 \alpha}(x)$ has a unique fixed point, then $\bar{x}$ is a GAS steady state of system (3).

The rest of the section is dedicated to the step-by-step construction of the proof of Theorem 1. However, due to the lack of space, the proofs are not deeply detailed.

Some properties about functions $F_{i \alpha}(x)$ are given:

Proposition 1: $\forall i \in\{1, \ldots, N\}, F_{i \alpha}(x)$ is strictly monotonically increasing and $\bar{x}_{i}$ is a fixed point of both $S_{i \alpha}(x)$ and $F_{i \alpha}(x)$. Moreover, if there exists a $j \in\{1, \ldots, N\}$ such that $F_{j \alpha}(x)$ has a unique fixed point, then $\forall i \in\{1, \ldots, N\} F_{i \alpha}(x)$ has a unique fixed point as well, and this fixed point is $\bar{x}_{i}$.

Bounds of the state space are now successively defined:

Definition 2:

- $x_{1 \text { max }}^{1}=M_{1}$,

- $x_{\text {imax }}^{1}=H_{i}\left(x_{i-1 \max }^{1}\right) \forall i \in\{2, \ldots, N\}$,

- $x_{1 \text { min }}^{1}=H_{1 \alpha}\left(x_{\text {Nmax }}^{1}\right)$,

- $x_{\text {imin }}^{1}=H_{i}\left(x_{i-1 \text { min }}^{1}\right) \forall i \in\{2, \ldots, N\}$,

where $M_{1}=H_{1 \alpha}(x=0)=\left(\kappa_{01}+\kappa_{1}\left(1+\alpha \bar{x}_{1}\right)\right) /\left(\gamma_{1}+\alpha \kappa_{1}\right)$ is the upper bound of $H_{1 \alpha}(x)$. Then by induction $\forall j>1$, $j \in \mathbb{N}$ :

- $x_{1 \max }^{j}=H_{1 \alpha}\left(x_{N \min }^{j-1}\right)$,

- $x_{\text {imax }}^{j}=H_{i}\left(x_{i-1 \text { max }}^{j}\right) \forall i \in\{2, \ldots, N\}$,

- $x_{1 \text { min }}^{j}=H_{1 \alpha}\left(x_{N \max }^{j}\right)$,

- $x_{\text {imin }}^{j}=H_{i}\left(x_{i-1 \text { min }}^{j}\right) \forall i \in\{2, \ldots, N\}$.

With the functions introduced in Definition 1, it is easy to prove that the previous bounds can also be defined as:

Proposition 2: $\forall j \in \mathbb{N}^{*}$, and $\forall i \in\{1, \ldots, N\}: x_{\text {imax }}^{j+1}=$ $F_{i \alpha}\left(x_{\text {imax }}^{j}\right)$ and $x_{\text {imin }}^{j+1}=F_{i \alpha}\left(x_{\text {imin }}^{j}\right)$.

Next proposition gives convergence results about sequences defined through functions $F_{i \alpha}$ :

Proposition 3: If $F_{1 \alpha}(x)$ has a unique fixed point, then $\forall i \in\{1, \ldots, N\}$, the sequence defined by $x_{i \max }^{j+1}=F_{i \alpha}\left(x_{\text {imax }}^{j}\right)$ with initial term $x_{i \max }^{1}$ (resp. $x_{i \min }^{j+1}=F_{i \alpha}\left(x_{i \min }^{j}\right)$ with initial term $x_{i m i n}^{1}$ ) is strictly monotonically decreasing (resp. increasing) and converges towards $\bar{x}_{i}$. As a consequence, $\forall i \in\{1, \ldots, N\}, x_{\text {imin }}^{j}<\bar{x}_{i}<x_{\text {imax }}^{j} \forall j \in \mathbb{N}^{*}$.

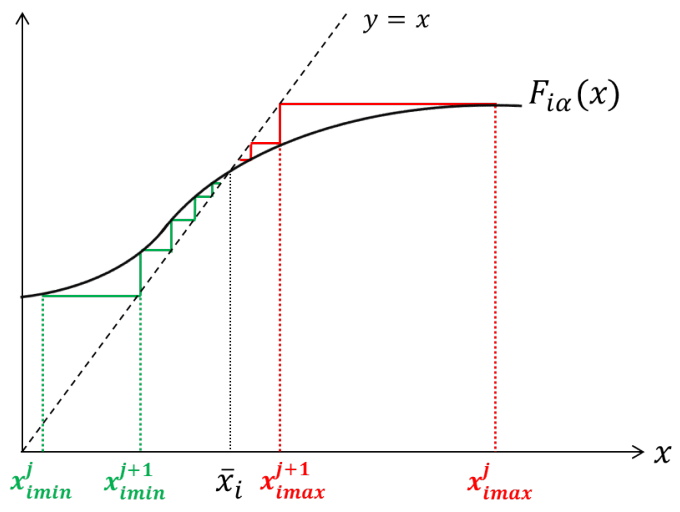

Fig. 2. Illustration of iterations for both sequences: $x_{i \max }^{j+1}=F_{i \alpha}\left(x_{\text {imax }}^{j}\right)$ (in red) with initial term $x_{i \max }^{1}$ and $x_{i \min }^{j+1}=F_{i \alpha}\left(x_{i \min }^{j}\right)$ (in green) with initial term $x_{\text {imin }}^{1}$, if $F_{1 \alpha}(x)$ has a unique fixed point. The two sequences converge towards $\bar{x}_{i}$, with $x_{\text {imax }}^{j}$ decreasing and $x_{\text {imin }}^{j}$ increasing.

This proposition, illustrated in Fig. 2, is easily proved by using the monotonic properties of $F_{i \alpha}(x) \forall i \in\{1, \ldots, N\}$. These sequences of boundaries shape the repelling hyperrectangles mentioned for the methodology:

Definition 3: $\mathscr{R}^{0}=\left\{x \mid x_{i} \geq 0 \forall i \in\{1, \ldots, N\}\right\}$ and $\forall j \in$ $\mathbb{N}^{*}, \mathscr{R}^{j}=\left\{x \mid x_{\text {imin }}^{j} \leq x_{i} \leq x_{\text {imax }}^{j} \forall i \in\{1, \ldots, N\}\right\}$.

These hyperrectangles are illustrated in Fig. 3. They have four principal interesting properties:

Proposition 4: If $F_{1 \alpha}(x)$ has a unique fixed point, then: (1) $\forall j \in \mathbb{N}, \bar{x} \in \mathscr{R}^{j}$,

(2) all these hyperrectangles are nested: $\forall j \in \mathbb{N}, \mathscr{R}^{j+1} \subset \mathscr{R}^{j}$ and $\mathscr{R}^{j+1} \neq \mathscr{R}^{j}$,

(3) $\forall j \in \mathbb{N}, \mathscr{R}^{j}$ is invariant,

(4) the hyperrectangles are successively repelling: $\forall j \in \mathbb{N}$ and for all initial condition $x_{0}=x(t=0) \in \mathscr{R}^{j}, \exists 0 \leq T^{j}<$ $+\infty$ such that $x(t) \in \mathscr{R}^{j+1} \forall t \geq T^{j}$.

Proof: The first and the second statements are straightforward with Proposition 3. The third invariant property means that as soon as a trajectory starts in a hyperrectangle, it cannot leave it. In order to prove it, it is shown that the vector field in each border of a hyperrectangle points inwards. The fourth proposition means that if a trajectory is in hyperrectangle $\mathscr{R}^{j}$, it will eventually reach the hyperrectangle $\mathscr{R}^{j+1}$ in a finite time. In order to prove it, it is possible to successively show that $\forall i \in\{1, \ldots, N\}$, the $x_{i}$-vector field in $\mathscr{R}^{j}$ points towards $\mathscr{R}^{j+1}$.

These four statements are illustrated in Fig. 3. From the construction of the hyperrectangles, next proposition is straightforward:

Proposition 5: If $F_{1 \alpha}(x)$ has a unique fixed point, then for any $\varepsilon>0$ and its associated ball $\mathscr{B}_{\varepsilon}=\left\{x \mid\|x-\bar{x}\|_{\infty} \leq \varepsilon\right\}$, it is possible to find $p_{\varepsilon} \in \mathbb{N}^{*}$ such that $\mathscr{R}^{j} \subset \mathscr{B}_{\varepsilon} \forall j \geq p_{\varepsilon}$.

This proposition simply means that, given a ball around $\bar{x}$, it is always possible to find its biggest embedded hyperrectangle. Besides, the infinite norm can be replaced by any 

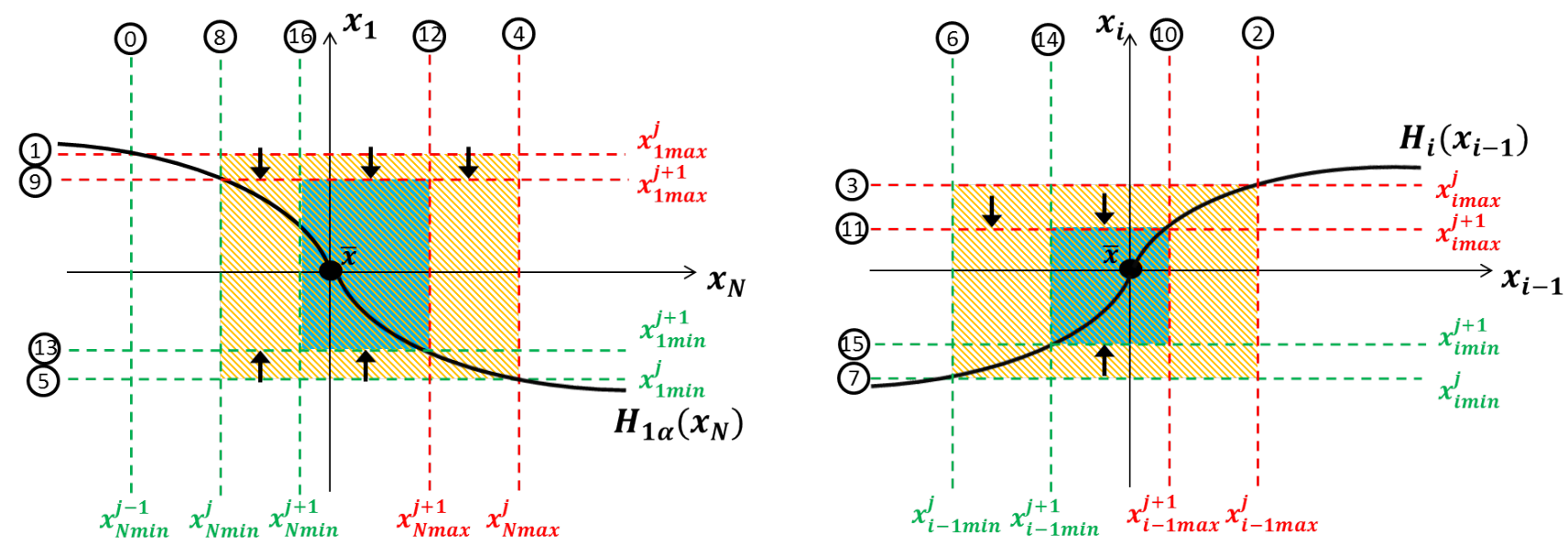

Fig. 3. These two schemes are an illustration of the construction of the successive hyperrectangles. The numbers from 1 to 8 explain the induction steps for the construction of the bounds defining $\mathscr{R}^{j}$ (represented by orange rectangles), and the numbers from 9 to 16 explain the induction steps for the construction of the bounds defining $\mathscr{R}^{j+1}$ (represented by blue rectangles). The vector field at the borders of $\mathscr{R}^{j}$ and $\mathscr{R}^{j+1}$ points inward, illustrating the invariant property in Proposition 4 . Moreover, by induction, the black arrows show that any trajectory starting in $\mathscr{R}^{j}$ arrives in finite time in $\mathscr{R}^{j+1}$, illustrating the repellent property in Proposition 4.

other norm. Finally, thanks to all the previous definitions and propositions, the final proof for Theorem 1 can be developed:

Proof: First, the global convergence is proved: let $\varepsilon>0$ and the associated ball $\mathscr{B}_{\varepsilon}=\left\{x \mid\|x-\bar{x}\|_{\infty} \leq \varepsilon\right\}$. From Proposition 5, $\exists p_{\varepsilon} \in \mathbb{N}^{*}$ such that $\mathscr{R}^{j} \subset \mathscr{B}_{\varepsilon} \forall j \geq p_{\varepsilon}$. Let $x_{0}=x(t=0) \in \mathbb{R}_{N}^{+}$an initial condition different from $\bar{x}$. From Definition 3, $\exists j_{0} \in \mathbb{N}$ such that $x_{0} \in \mathscr{R}^{j} \forall j \leq j_{0}, j \in \mathbb{N}$, and $x_{0} \notin \mathscr{R}^{j} \forall j>j_{0}$. According to the fourth statement in Proposition 4, $\exists 0 \leq T^{j_{0}}<+\infty$ such that $x(t) \in \mathscr{R}^{j_{0}+1} \forall t \geq$ $T^{j_{0}}$. By induction, let $T_{\varepsilon}=\sum_{k=j_{0}}^{p_{\varepsilon}-1} T^{k}$ where $T^{k}$ are defined in the fourth statement of Proposition 4. Then, $\forall t \geq T_{\varepsilon}$, $x(t) \in \mathscr{R}^{p_{\varepsilon}}$. Then from Proposition $5, \forall t \geq T_{\varepsilon}, x(t) \in \mathscr{B}_{\varepsilon}$. In conclusion, it has been shown that $\forall x_{0}=x(t=0) \in \mathbb{R}_{N}^{+}$ and $\forall \varepsilon>0, \exists 0 \leq T_{\varepsilon}<+\infty$ such that $\forall t \geq T_{\varepsilon}, x(t) \in \mathscr{B}_{\varepsilon}$. This is the definition of global convergence.

Now, the Lyapunov stability is proved: let $\delta>0$ and the associated ball $\mathscr{B}_{\delta}=\left\{x \mid\|x-\bar{x}\|_{\infty} \leq \delta\right\}$. From Proposition 5, $\exists p_{\delta} \in \mathbb{N}^{*}$ such that $\mathscr{R}^{j} \subset \mathscr{B}_{\delta} \forall j \geq p_{\delta}$. From the third statement in Proposition $4, \mathscr{R}^{p}$ is invariant. Then, $\forall x_{0}=x(t=0) \in \mathscr{R}^{p_{\delta}}, x(t) \in \mathscr{R}^{p_{\delta}} \forall t \geq 0$. Finally, as $\mathscr{R}^{p} \subset \mathscr{B}_{\delta}, x(t) \in \mathscr{B}_{\delta} \forall t \geq 0$. This is the definition of Lyapunov stability.

In conclusion, it has been shown in this section that if the function $F_{1 \alpha}(x)$ has a unique fixed point, then the steady state $\bar{x}$ of system (3) is GAS. In next section, the conditions on $\alpha$ will be given in order to ensure that $F_{1 \alpha}(x)$ has a unique fixed point.

\section{APPLICATION}

\section{A. Functions with negative Schwarzian derivatives}

As the number of fixed points of a function is directly related to its shape, a shape-indicator of $F_{1 \alpha}(x)$, called "Schwarzian derivative" [1], is calculated.
Definition 4: The Schwarzian derivative of a function $f(x)$, denoted by $S f(x)$, is equal to:

$$
\left\{\begin{array}{l}
S f(x)=-\infty \quad \text { if } \quad f^{\prime}(x)=0 \\
S f(x)=\frac{f^{(3)}(x)}{f^{\prime}(x)}-\frac{3}{2}\left(\frac{f^{\prime \prime}(x)}{f^{\prime}(x)}\right)^{2} \quad \text { if } \quad f^{\prime}(x) \neq 0 .
\end{array}\right.
$$

With basic calculations, it is easy to show that:

Property 3: $F_{1 \alpha}(x)$ has a negative Schwarzian derivative.

The sign of the Schwarzian derivative of a function gives relevant information about the shape of this function:

Property 4: $F_{1 \alpha}(x)$ is a sigmoid function.

Proof: It is easy to show that $\left.F_{1 \alpha}{ }^{\prime}(x)>0 \forall x \in\right] 0,+\infty[$, $F_{1 \alpha}{ }^{\prime}(0)=0$ from the properties of Hill functions, and $F_{1 \alpha}(x)$ is bounded. It follows that there exists $a>0$ such that $F_{1 \alpha}{ }^{\prime}(x)$ is strictly increasing $\left.\forall x \in\right] 0, a\left[\right.$ and $F_{1 \alpha}{ }^{\prime}(x)$ is strictly decreasing $\forall x \in] a,+\infty[$ (see [1] for the details). This is exactly the definition of a sigmoid function.

This sigmoidal shape is essential in order to determine the number of fixed points of $F_{1 \alpha}(x)$ :

Property 5: $F_{1 \alpha}(x)$ cannot have more than three fixed points, and among them is $\bar{x}_{1}$. If there are three fixed points, $\bar{x}_{1}$ is the middle one. Moreover, $\bar{x}_{1}$ is the unique fixed point of $F_{1 \alpha}(x)$ if and only if $F_{1 \alpha}{ }^{\prime}\left(\bar{x}_{1}\right)<1$.

This result can be proved thanks to the properties of sigmoid functions, and is illustrated in Fig. 4.

The conclusion of this section is the following: if there exist conditions on $\alpha$ such that $F_{1 \alpha}{ }^{\prime}\left(\bar{x}_{1}\right)<1$, then Property 5 ensures $F_{1 \alpha}(x)$ to have a unique fixed point, and Theorem 1 guarantees $\bar{x}$ to be GAS. Next section investigates these conditions on $\alpha$. 

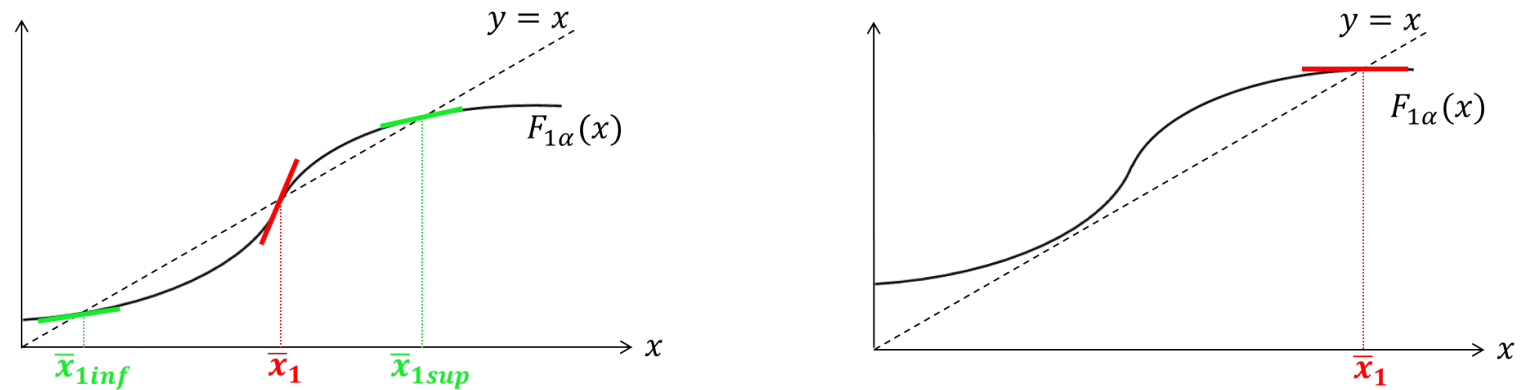

Fig. 4. For both plots, the thick black line is the sigmoid function $F_{1 \alpha}(x)$. The intersections with $y=x$ (represented by the dashed black line) are illustrated. Left: $F_{1 \alpha}(x)$ has three fixed points, leading to $F_{1 \alpha}{ }^{\prime}\left(\bar{x}_{1}\right)>1$. Right: $F_{1 \alpha}(x)$ has a unique fixed point $\bar{x}_{1}$, leading to $F_{1 \alpha}{ }^{\prime}\left(\bar{x}_{1}\right)<1$.

\section{B. Conditions on $\alpha$}

This section proves that there exist conditions on $\alpha$ such that the steady state $\bar{x}$ of system (3) becomes GAS.

Lemma 1: If $\alpha>-\gamma_{1}\left(S_{1}^{\prime}\left(\bar{x}_{1}\right)+1\right) /\left(\kappa_{1} \mathrm{~h}^{-}\left(\bar{x}_{N}, \theta_{N}, n_{N}\right)\right)=$ $\alpha_{0}$, where $S_{1}(x)=H_{1} \circ H_{N} \circ H_{N-1} \circ \ldots \circ H_{3} \circ H_{2}(x)$ is defined in section II, then $F_{1 \alpha}{ }^{\prime}\left(\bar{x}_{1}\right)<1$.

Proof: From the results presented in section III, the derivative of $F_{1 \alpha}(x)$ is calculated:

$$
\left.F_{1 \alpha^{\prime}}{ }^{\prime} \bar{x}_{1}\right)=S_{1 \alpha^{\prime}}\left(S_{1 \alpha}\left(\bar{x}_{1}\right)\right) S_{1 \alpha^{\prime}}\left(\bar{x}_{1}\right)=\left(S_{1 \alpha^{\prime}}\left(\bar{x}_{1}\right)\right)^{2} .
$$

As $S_{1 \alpha^{\prime}}(x) \leq 0 \forall x \geq 0$, then $F_{1 \alpha^{\prime}}\left(\bar{x}_{1}\right)<1 \Longleftrightarrow S_{1 \alpha^{\prime}}\left(\bar{x}_{1}\right)>$ -1 . The derivative of $S_{1 \alpha}\left(\bar{x}_{1}\right)$ is calculated:

$$
S_{1 \alpha^{\prime}}\left(\bar{x}_{1}\right)=H_{1 \alpha^{\prime}}\left(\bar{x}_{N}\right) H_{N}{ }^{\prime}\left(\bar{x}_{N-1}\right) \ldots H_{3}{ }^{\prime}\left(\bar{x}_{2}\right) H_{2}{ }^{\prime}\left(\bar{x}_{1}\right) .
$$

In this last equation, everything is fixed, except the term $H_{1} \alpha^{\prime}\left(\bar{x}_{N}\right)$ that depends on $\alpha$. It is important to notice that for $\alpha=0$, then $S_{1 \alpha}(x)=S_{1}(x)$, where $S_{1}(x)$ is the function defined for the uncontrolled system (1) in section II. From the properties of system (1), it has been shown that $S_{1}^{\prime}(x) \leq 0$ and by hypothesis it is considered that system (1) shows undesired oscillations. As a consequence, the unique steady state $\bar{x}$ of system (1) is unstable. Hence, the condition $\left(S_{1} \circ S_{1}\right)^{\prime}\left(\bar{x}_{1}\right)<1$ cannot hold. Indeed, if $\left(S_{1} \circ S_{1}\right)^{\prime}\left(\bar{x}_{1}\right)<1$ was true, then by applying Property 5 and Theorem 1, the steady state $\bar{x}$ of system (1) would be GAS. It follows that $\left(S_{1} \circ S_{1}\right)^{\prime}\left(\bar{x}_{1}\right)>1$. Moreover, $\left(S_{1} \circ S_{1}\right)^{\prime}\left(\bar{x}_{1}\right)=S_{1}^{\prime}\left(\bar{x}_{1}\right)^{2}>1$. As $S_{1}^{\prime}(x) \leq 0, S_{1}^{\prime}\left(\bar{x}_{1}\right)<-1$. Then, for $\alpha=0, S_{1 \alpha}{ }^{\prime}\left(\bar{x}_{1}\right)=S_{1}^{\prime}\left(\bar{x}_{1}\right)<$ -1 . Now, the influence of $\alpha$ on the derivative $S_{1 \alpha^{\prime}}\left(\bar{x}_{1}\right)$ is investigated. From the definition of $H_{1}(x)$ given in section II, $H_{1}{ }^{\prime}\left(\bar{x}_{N}\right)=\frac{\kappa_{1}}{\gamma_{1}} \frac{\partial \mathrm{h}^{-}\left(\bar{x}_{N}, \theta_{N}, n_{N}\right)}{\partial x}$. Hence, the function $S_{1 \alpha^{\prime}}{ }^{\prime}\left(\bar{x}_{1}\right)$ can be written:

$$
S_{1 \alpha^{\prime}}^{\prime}\left(\bar{x}_{1}\right)=\gamma_{1} \frac{\alpha\left(\gamma_{1} \bar{x}_{1}-\kappa_{01}\right)+\gamma_{1}}{\left(\gamma_{1}+\alpha \kappa_{1} \mathrm{~h}^{-}\left(\bar{x}_{N}, \theta_{N}, n_{N}\right)\right)^{2}} S_{1}^{\prime}\left(\bar{x}_{1}\right) .
$$

From the definition of the steady state of the uncontrolled $\operatorname{system}(1), \kappa_{01}+\kappa_{1} \mathrm{~h}^{-}\left(\bar{x}_{N}, \theta_{N}, n_{N}\right)-\gamma_{1} \bar{x}_{1}=0$ which is equivalent to $\gamma_{1} \bar{x}_{1}-\kappa_{01}=\kappa_{1} \mathrm{~h}^{-}\left(\bar{x}_{N}, \theta_{N}, n_{N}\right)$. This relation is used in the previous equation, leading to:

$$
S_{1 \alpha^{\prime}}^{\prime}\left(\bar{x}_{1}\right)=\frac{\gamma_{1}}{\gamma_{1}+\alpha \kappa_{1} \mathrm{~h}^{-}\left(\bar{x}_{N}, \theta_{N}, n_{N}\right)} S_{1}^{\prime}\left(\bar{x}_{1}\right) .
$$

Now the derivative of $S_{1 \alpha^{\prime}}\left(\bar{x}_{1}\right)$ with respect to $\alpha$ is calculated:

$$
\frac{\partial S_{1 \alpha^{\prime}}\left(\bar{x}_{1}\right)}{\partial \alpha}=-\gamma_{1} S_{1}^{\prime}\left(\bar{x}_{1}\right) \frac{\kappa_{1} \mathrm{~h}^{-}\left(\bar{x}_{N}, \theta_{N}, n_{N}\right)}{\left(\gamma_{1}+\alpha \kappa_{1} \mathrm{~h}^{-}\left(\bar{x}_{N}, \theta_{N}, n_{N}\right)\right)^{2}} .
$$

Finally, for $\alpha \geq 0$, as $S_{1}^{\prime}\left(\bar{x}_{1}\right) \leq 0$, then $\frac{\partial S_{1 \alpha^{\prime}}\left(\bar{x}_{1}\right)}{\partial \alpha} \geq 0$. Then, the derivative $S_{1 \alpha}{ }^{\prime}\left(\bar{x}_{1}\right)$ is an increasing function of $\alpha$. Moreover, it is easy to show that $\lim _{\alpha \rightarrow+\infty} S_{1 \alpha^{\prime}}\left(\bar{x}_{1}\right)=0$, and it has been explained previously that when $\alpha=0, S_{1 \alpha^{\prime}}\left(\bar{x}_{1}\right)=S_{1}^{\prime}\left(\bar{x}_{1}\right)<$ -1 . Finally, the function $S_{1 \alpha^{\prime}}\left(\bar{x}_{1}\right)$ is an increasing function of $\alpha$ that is strictly smaller than -1 when $\alpha=0$ and that tends to 0 when $\alpha$ tends to infinity. Then, there exists a value of $\alpha$ called $\alpha_{0}$ such that $\forall \alpha>\alpha_{0}, S_{1 \alpha}^{\prime}\left(\bar{x}_{1}\right)>-1$. This $\alpha_{0}$ is determined by solving $S_{1 \alpha}^{\prime}\left(\bar{x}_{1}\right)=-1$ :

$$
S_{1 \alpha}{ }^{\prime}\left(\bar{x}_{1}\right)=-1 \Longleftrightarrow \alpha=\frac{-\gamma_{1}\left(S_{1}^{\prime}\left(\bar{x}_{1}\right)+1\right)}{\kappa_{1} \mathrm{~h}^{-}\left(\bar{x}_{N}, \theta_{N}, n_{N}\right)} .
$$

Again, by hypothesis $S_{1}^{\prime}\left(\bar{x}_{1}\right)<-1$, hence $S_{1}^{\prime}\left(\bar{x}_{1}\right)+1<0$. Finally $\alpha_{0}=-\gamma_{1}\left(S_{1}^{\prime}\left(\bar{x}_{1}\right)+1\right) /\left(\kappa_{1} \mathrm{~h}^{-}\left(\bar{x}_{N}, \theta_{N}, n_{N}\right)\right)>0$. The proof is now completed: when $\alpha>\alpha_{0}, F_{1} \alpha^{\prime}\left(\bar{x}_{1}\right)<1$.

Lemma 1 allows the statement of the main result:

Theorem 2: If $\alpha>\alpha_{0}$, then $\bar{x}$ is a GAS steady state of system (3).

Proof: If $\alpha>\alpha_{0}$, then with Lemma $1, F_{1 \alpha^{\prime}}\left(\bar{x}_{1}\right)<1$. It follows from Property 5 that $F_{1 \alpha}(x)$ has a unique fixed point. Theorem 1 ends the proof.

As an example, Fig. 5 presents a simulation of a negative feedback loop of dimension 3. As explained in the introduction, without control, this loop was constructed in order to show undesired sustained oscillations. With the affine control meeting the condition $\alpha>\alpha_{0}$, the simulation shows global convergence towards the unique steady state of this 3-dimensional system, as analytically proved.

\section{CONCLUSIONS AND FUTURE WORKS}

\section{A. Conclusions}

In this paper it has been shown that with an affine control, a negative feedback loop that presents undesired oscillations can be stabilized. Indeed, for a control parameter $\alpha$ chosen large enough, the unique steady state of the negative feedback loop becomes GAS. Due to the non-linearities of this 

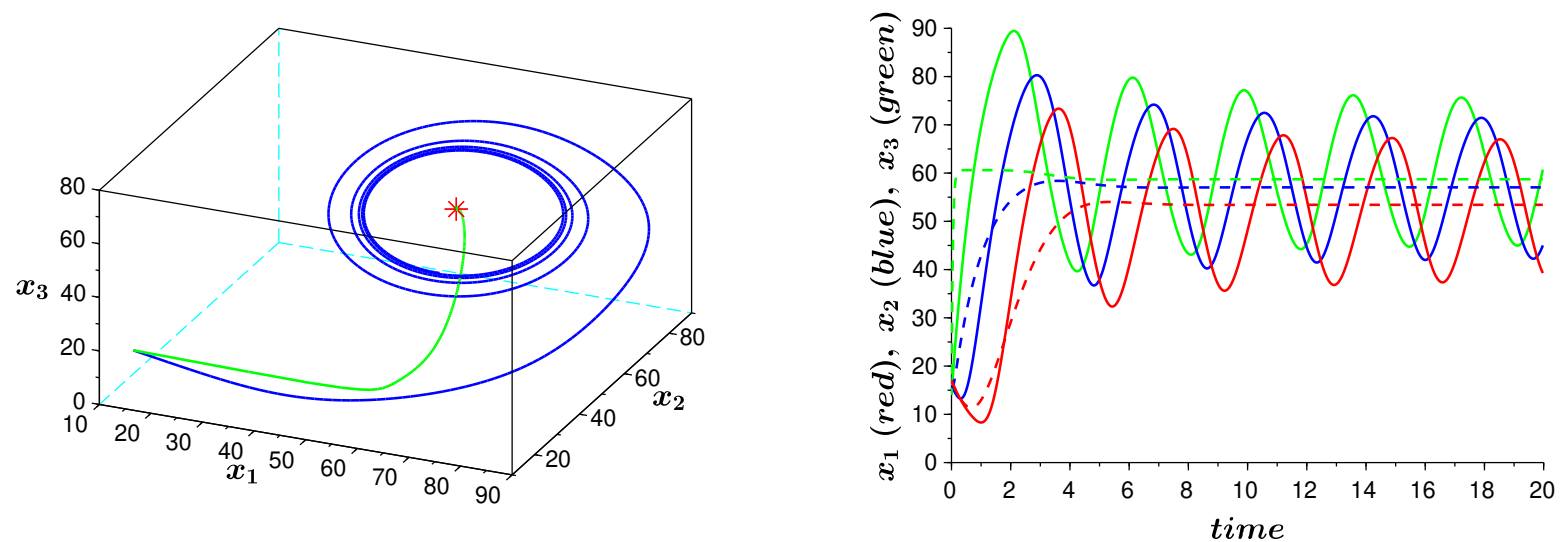

Fig. 5. For both plots, the parameters are: $\kappa_{0 i}=2, \kappa_{i}=100, \gamma_{i}=1, \theta_{i}=56.39$ and $n_{i}=5, \forall i \in\{1,2,3\}$, leading to $\bar{x}=(58.7,57,53.4)$. The initial condition for the simulation is $x_{0}=(14,16,17)$. With these parameters, $\alpha_{0}=0.17$, and $\alpha$ is fixed to $\alpha=0.22$. Left: trajectories in the state space. The steady state is represented by the red star. The blue line is a simulation of system (1) with initial condition $x_{0}$ and converges towards a periodic orbit. The green line is a simulation of system (3) with same initial condition $x_{0}$ and converges towards $\bar{x}$. Right: same trajectory as the left plot, but against time. The plain (resp. dashed) lines are without (resp. with) control.

controlled system, a new methodology has been developed in order to obtain global results. A nested sequence of repelling hyperrectangles has been constructed and has been shown to act as Lyapunov function level-sets. The affine control law presented in this paper is only dependent on the first variable $x_{1}$ and only acts on its expression. It is interesting to note that this simple control law is able to stabilize a whole system in any dimension $N$. Moreover, from a biological point of view, this control law minimizes the experiment set-up complexity. On the one hand, only the first gene must be measured: this is convenient as measurements in biology are usually tedious. On the other hand, the control only impacts the interaction between the last gene and the first gene. Again, this suggests that only one control device is enough, simplifying the experiment.

\section{B. Future Works}

Interestingly, the method explained in this work seems valid in a more general context. For a general negative loop system, if the nullclines are strictly monotonic and partition the space in two distinct regions in which the vector field has opposite sign, then Theorem 1 seems to apply. This analytic result is a natural extension of this work.

A couple of remarks can be given regarding a possible biological implementation. Firstly, as mentioned, the control may take negative values. From a biological point of view, this means that the control device must be able to either influence the production of the first molecule $x_{1}$ (when $u\left(x_{1}\right)>0$ ) or degrade it (when $u\left(x_{1}\right)<0$ ). In the first case, the biological tools mentioned in the introduction may allow the application of this control. However, for a negative control, the biological implementation does not seem straightforward. This situation may be avoided if the control parameter $\alpha$ can be chosen small enough. In this case, the control law stays positive in the invariant bounded region of the state space. If this is not possible, the control law may be saturated: the tools developed in this paper for the global convergence may be easily adapted in this new context. Secondly, this classical control technique depends on precise knowledge of the state space (namely $x_{1}$ ). However, most of the time the measurements available in biology are of qualitative nature leading to partially known systems. Another interesting extension of this work may be to consider piecewise linear control laws.

Finally, this study can be extended in order to control biological oscillations. Indeed, disorders in biological rhythms, such as the circadian clock, are known to provoke various diseases. A similar control law may be designed in order to tune the phase and the amplitude of the system.

\section{REFERENCES}

[1] M. E. Ahsen, H. Özbay, and S.-I. Niculescu. Analysis of deterministic cyclic gene regulatory network models with delays. Birkhäuser, 2015.

[2] D. J. Allwright. A global stability criterion for simple control loops. J. Math. Biology, 4:363-373, 1977.

[3] D. Angeli and E. D. Sontag. Monotone control systems. IEEE Transactions on automatic control, 48(10):1684-1698, 2003.

[4] B. d'Andréa Novel and M. De Lara. Control theory for engineers. Springer, 2013.

[5] B. C. Goodwin et al. Temporal organization in cells. A dynamic theory of cellular control processes. London and New York: Academic Press., 1963.

[6] J. B. Lugagne, S. Sosa Carrillo, M. Kirch, A. Köhler, G. Batt, and P. Hersen. Balancing a genetic toggle switch by real-time feedback control and periodic forcing. Nature Communications, 8:1671, 2017.

[7] A. Milias-Argeitis, M. Rullan, S. K. Aoki, P. Buchmann, and M. Khammash. Automated optogenetic feedback control for precise and robust regulation of gene expression and cell growth. Nature communications, 7:12546, 2016.

[8] R. Milo, S. Shen-Orr, S. Itzkovitz, N. Kashtan, D. Chklovskii, and U. Alon. Network motifs: Simple building blocks of complex networks. Science, 298:824-827, Oct 2002.

[9] H. L. Smith. Monotone dynamical systems: an introduction to the theory of competitive and cooperative systems. Number 41. American Mathematical Soc., 2008.

[10] A. Szybińska and W. Leśniak. P53 dysfunction in neurodegenerative diseases-the cause or effect of pathological changes? Aging and disease, 8(4):506, 2017.

[11] J. Uhlendorf, A. Miermont, T. Delaveau, G. Charvin, F. Fages, S. Bottani, G. Batt, and P. Hersen. Long-term model predictive control of gene expression at the population and single-cell levels. Proceedings of the National Academy of Sciences, 109(35):14271-14276, August 2012. 